\title{
Effects of Parametrical and Trial-to-Trial Variation in Prior Probability Processing Revealed by Simultaneous Electroencephalogram/Functional Magnetic Resonance Imaging
}

\author{
Christina Scheibe, ${ }^{1,2,3}$ Markus Ullsperger, ${ }^{4,5}$ Werner Sommer, ${ }^{3}$ and Hauke R. Heekeren ${ }^{1,2,6}$ \\ ${ }^{1}$ Neuroscience Research Center and Berlin Neuroimaging Center, Charité University Hospital, D-10117 Berlin, Germany, ${ }^{2}$ Max Planck Institute for Human \\ Development, D-14195 Berlin, Germany, ${ }^{3}$ Department of Psychology, Humboldt University of Berlin, D-10099 Berlin, Germany, ${ }^{4}$ Max Planck Institute for \\ Neurological Research, D-50931 Cologne, Germany, ${ }^{5}$ Donders Institute for Brain, Cognition, and Behaviour, Radboud University Nijmegen, 6525 EN \\ Nijmegen, The Netherlands, and 'Department of Psychology and Education, Freie Universität Berlin, D-14195 Berlin, Germany
}

Prior knowledge of the probabilities concerning decision alternatives facilitates the selection of more likely alternatives to the disadvantage of others. The neural basis of prior probability (PP) integration into the decision-making process and associated preparatory processes is, however, still essentially unknown. Furthermore, trial-to-trial fluctuations in PP processing have not been considered thus far. In a previous study, we found that the amplitude of the contingent negative variation (CNV) in a precueing task is sensitive to PP information (Scheibe et al., 2009). We investigated brain regions with a parametric relationship between neural activity and PP and those regions involved in PP processing on a trial-to-trial basis in simultaneously recorded electroencephalographic (EEG) and functional magnetic resonance imaging (fMRI) data. Conventional fMRI analysis focusing on the information content of the probability precue revealed increasing activation of the posterior medial frontal cortex with increasing PP, supporting its putative role in updating action values. EEG-informed fMRI analysis relating single-trial CNV amplitudes to the hemodynamic signal addressed trial-to-trial fluctuations in PP processing. We identified a set of regions mainly consisting of frontal, parietal, and striatal regions that represents unspecific response preparation on a trial-to-trial basis. A subset of these regions, namely, the dorsolateral prefrontal cortex, the inferior frontal gyrus, and the inferior parietal lobule, showed activations that exclusively represented the contributions of PP to the trial-to-trial fluctuations of the CNV.

\section{Introduction}

The neural basis of perceptual decision making comprises the accumulation and integration of sensory evidence in specific brain areas (Mazurek et al., 2003; Heekeren et al., 2004, 2008; Gold and Shadlen, 2007). Prior knowledge of the probability concerning the decision alternatives is integrated into the decision-making process and facilitates the selection of probable alternatives to the disadvantage of others (Glimcher, 2001; Opris and Bruce, 2005; Gold and Shadlen, 2007). At the behavioral level, prior probability (PP) modulates response time (RT) (Carpenter and Williams, 1995; Reddi and Carpenter, 2000; Reddi et al., 2003; Carpenter, 2004). The neural basis of PP integration into the decision-making process and associated preparatory processes is, however, still essentially unknown.

Received Aug. 12, 2009; revised Sept. 29, 2010; accepted Sept. 30, 2010.

This research was supported by the Max Planck Society and by grants from the Graduate Program Berlin (Scholarship NaFöG) and German Research Foundation Grant HE 3347/2-1. We thank the technical staff of the Max Planck Institute for Human Cognitive and Brain Sciences (Leipzig, Germany) for support in data collection.

Correspondence should be addressed to Christina Scheibe, Department of Neurology, Charité Campus Mitte, Charitéplatz 1, 10117 Berlin, Germany. E-mail: christina.scheibe@charite.de.

DOI:10.1523/JNEUROSCI.3949-09.2010

Copyright $\odot 2010$ the authors $\quad$ 0270-6474/10/3016709-09\$15.00/0
In a previous study using electroencephalography (EEG), we found evidence for the integration of PP into the decision-making process at a premotor stage (Scheibe et al., 2009). Specifically, higher-order premotor preparation processes were parametrically modulated by probability information as indicated by the contingent negative variation ( $\mathrm{CNV})$, a sustained negative potential shift that is maximal at central electrode positions and develops after a precue followed by a second stimulus (Walter et al., 1964). In our study, a precue provided preliminary probabilistic information about the decision demanded by a second stimulus. Mean CNV amplitude increases parametrically with increasing PP. In addition, a dipole source analysis indicated a locus of parametrically modulated activity during the preparation phase in the anterior cingulate cortex (Scheibe et al., 2009). Brain imaging methods with higher spatial resolution, such as functional magnetic resonance imaging (fMRI), have not yet been applied to verify this notion [but see a few EEG-fMRI studies using oddball tasks and RT or latency-based analysis (Bénar et al., 2007; Goldman et al., 2009) ]. Furthermore, our previous findings rely on the mean CNV amplitude while trial-to-trial variability is not taken into account. Considering the parametrical influence of PP on the mean CNV amplitude, the single-trial amplitude of the CNV 
might contain additional information about PP processing and interactions with ongoing brain activity. Recent studies suggest that ongoing fluctuations in several event-related brain potentials (ERPs) are not random noise but instead reflect specific intrinsic and extrinsic processes (Debener et al., 2005, 2006; Eichele et al., 2005). Trial-totrial fluctuations might represent dynamic modulations of intrinsic probabilistic models of anticipated events (Fox et al., 2006; Mars et al., 2008) or fluctuations of attention (Weissman et al., 2006; Goldman et al., 2009) and effort-related decision making (Mulert et al., 2008; Esposito et al., 2009).

The goal of the present study was to identify the brain regions in which neural activity is parametrically modulated by $\mathrm{PP}$, as well as those in which the blood oxygen level-dependent (BOLD) signal covaries with trial-to-trial fluctuations in PP processing and PP-based response preparation. To find regions that are parametrically modulated by PP, conventional fMRI analyses can be applied. In contrast, to identify brain regions in which activation underlies a trial-totrial fluctuation in PP processing, EEG and fMRI need to be combined. Therefore, we simultaneously recorded EEG and fMRI data and conducted a conventional as well as an EEGinformed fMRI analysis (for review, see Debener et al., 2006; Ullsperger, 2010). The latter procedure allows for relating singletrial CNV amplitudes directly to the BOLD signal.

\section{Materials and Methods}

Participants. Sixteen healthy volunteers ( 8 female, 8 male; mean \pm SD age, $26 \pm 2.83$ years) participated in the experiment. They were paid for their participation and gave written informed consent. All participants were right-handed (mean \pm SD handedness index, $97.4 \pm$ 4.7; Edinburgh Handedness Inventory) (Oldfield, 1971) and had normal or corrected-to-normal vision. The study was approved by the local Human Subjects Committee and adhered to the Human Subjects Guidelines of the Declaration of Helsinki.

Task and procedure. We adapted the number comparison task from Schwarz and Stein (1998) to meet our requirements. The same task was used in the previous EEG study (Scheibe et al., 2009). Task and temporal sequence of the stimuli in a trial are shown in Figure $1 \mathrm{~A}$. Each trial lasted $4500 \mathrm{~ms}$ and began with a color change of the fixation cross for $500 \mathrm{~ms}$, followed by the presentation of the first number (S1) to the left or right of the fixation cross (randomized across conditions). The foreperiod lasted $2000 \mathrm{~ms}$ and was defined as the time from the onset of S1 presentation to the onset of the presentation of the second number (S2). After the foreperiod, S2 was added to the display on the opposite side and demanded a response on the side of the numerically larger number. Both numbers remained on the screen until the participant responded by button press. Maximum time allowed for a response was $1200 \mathrm{~ms}$ after S2. If a button was pressed during the foreperiod, $>1200 \mathrm{~ms}$ after S2, or at the incorrect side, immediate feedback was delivered on the screen with the terms "too early," "too slow," or "error," respectively. No feedback was given after correct responses. To minimize eye movements, participants were instructed to focus on the fixation cross throughout the trial. Participants viewed the stimuli via a custom-made mirror system. They held a response key in each hand and performed button presses with their thumbs.

S1 had values of $1,3,5,7$, or 9; S2 had any integer value between 1 and 9 but never the same value as S1. S1 served as a task-inherent precue predicting the PP of the side with the larger number. The side with the larger number is predicted with PP of 1.0 by S1 values of 1 or 9, with PP
B

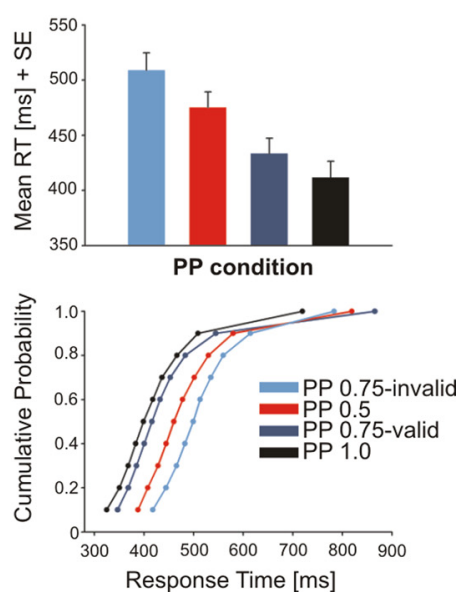


ing the entire brain. Head movement was minimized using a vacuum pad. A 200 ms no-acquisition period was included in each TR to allow online monitoring of the EEG recording. Between the runs, a 1 min rest was given while scanning continued. One-fifth of the total time of each run consisted of null events that were randomly inserted between trials. Trials were jittered in steps of $0.25 \mathrm{TR}$. The intertrial interval varied from 0 to $9500 \mathrm{~ms}$ (mean $\pm \mathrm{SD}, 1036 \pm 1194 \mathrm{~ms}$ ). The temporal sequence of the trials in each run was optimized using Optseq2 (http://surfer.nmr.mgh. harvard.edu/optseq). The first six scans were discarded to allow longitudinal magnetization to reach equilibrium and because they contained the practice trials.

The EEG was continuously recorded from 59 standard scalp sites using $\mathrm{Ag} / \mathrm{AgCl}$ electrodes and the Brain Amps MR Plus (Brain Products Inc.). Additional electrodes were placed below and above the right eye and on the lower back to monitor eye movements and the electrocardiogram, respectively, and were also referenced to FCz (cf. Debener et al., 2008).

Behavioral data analysis. RTs were analyzed using one-way repeatedmeasures ANOVAs with the factor PP condition (PP 1.0, PP 0.75-valid, PP 0.5, PP 0.75-invalid). Violation of sphericity was Greenhouse-Geisser corrected. Bonferroni's corrected pairwise $t$ tests were calculated to compare the mean RTs between PP conditions.

In principle, it is possible that participants prepared a response in only some trials, resulting in very fast responses in a few trials for high PPs and very slow responses for low PPs. In that case, RTs would differ between conditions only at very low and very high percentiles and not at intermediate percentiles. To ascertain that the PP effect on mean RT did not result from a small set of trials, we calculated vincentized cumulative probability distributions of RTs for all PP conditions (Miller, 1998). For additional analysis, we calculated a repeated-measures ANOVA with the factors PP condition (PP 0.5, PP 0.75-valid, PP 0.75-invalid, and PP 1.0) and decile (10 levels).

EEG data analysis. We removed the fMRI gradient artifact using an average artifact template procedure with a sliding average calculation, as implemented in the Brain Vision Analyzer software (Brain Products Inc.). A trigger from the MR system recorded with the EEG indicated the onset of the gradient artifact, and the data were corrected relative to this onset. The artifact template calculation was based on a sliding average with a width of 20 gradient artifacts.

EEG data were low-pass filtered at $40 \mathrm{~Hz}$, downsampled to $250 \mathrm{~Hz}$, exported to Matlab (MathWorks), and further analyzed using EEGLAB (Delorme and Makeig, 2004). The data were re-referenced to average reference. Practice trials and pauses were removed from the data. Also, periods with nonstereotyped artifacts (e.g., head movements, swallowing) as well as drifting or extremely noisy channels were removed manually. Ballistocardiogram artifacts attributable to pulse-rated movements of the scalp electrodes and electrically conductive blood were detected and removed using the algorithm implemented in EEGLAB [FMRIB (Oxford University Centre for Functional MRI of the Brain) plugin] (Niazy et al., 2005). Subsequently, we performed temporal infomax independent component analysis (ICA) (Bell and Sejnowski, 1995). Independent components with signal and scalp topography that could be assigned to known stereotyped artifacts (e.g., horizontal and vertical eye movements) were removed from the data (Jung et al., 2000a,b; Delorme et al., 2007). The remaining components were backprojected on the scalp to obtain EEG data with diminished contribution of artifacts. This ICA procedure for artifact removal has been used in a number of previous studies (Debener et al., 2005; Eichele et al., 2005).

We segmented the continuous EEG data into epochs of $3800 \mathrm{~ms}$ starting $300 \mathrm{~ms}$ before S1 and used the $300 \mathrm{~ms}$ interval before S1 for baseline correction. For the conventional analysis, all epochs were averaged over trials per PP condition (PP 0.5, PP 0.75, and PP 1.0) within each participant and across participants. The amplitude of the $\mathrm{CNV}$ was calculated as the mean voltage over $200 \mathrm{~ms}$ before S2. We used a repeated-measures ANOVA containing the factors PP condition (PP 0.5, PP 0.75, and PP 1.0) and electrode (47 electrodes) to compare average $\mathrm{CNV}$ amplitudes between conditions and electrodes. Because the CNV amplitude is maximal at midline electrode positions and can be hardly identified at lateral electrodes, we excluded the most lateral electrodes from the analysis and calculated the repeated-measures ANOVA using the remaining 47 electrodes (AF7, AF5, AFz, AF6, AF8, F7, F5, F3, Fz, F4, F6, F8, FT7, FC5, FC3, FCz, FC4, FC6, FT8, T7, C5, C3, Cz, C4, C6, T8, TP7, CP5, CP3, CP4, CP6 TP8, P7, P5, P3, Pz, P4, P6, P8, PO7, PO5, POz, PO6, PO8, O1, Oz, and $\mathrm{O} 2$ ). When the factor PP condition turned out to be significant, an additional repeated-measures ANOVA was conducted for the electrode with the most pronounced CNV. We calculated paired $t$ tests to compare CNV amplitudes within the factor PP condition.

For the trial-to-trial analysis, we calculated the CNV amplitude for each epoch as the mean voltage over $1000 \mathrm{~ms}$ before S2 at electrode position $\mathrm{Cz}$ (where we found the most pronounced $\mathrm{CNV}$ in the group analysis). We used an interval of $1000 \mathrm{~ms}$ for the CNV amplitude calculation to reduce the variance attributable to EEG fluctuations within single trials.

The association between $\mathrm{CNV}$ amplitude and RT was determined by a correlation between single-trial CNV amplitudes and RTs per participant. For group analysis, the resulting individual correlation coefficients were Fisher $z$-transformed to test them against zero by applying a onesample $t$ test. The resulting mean value was subsequently retransformed into a correlation coefficient. The same correlation analysis and subsequent $t$ test were calculated using single-trial CNV amplitude residuals after removing the variance accounted for the PP manipulation [see below, fMRI data analysis (model 3)], to examine whether CNV amplitude fluctuations unrelated to the use of information provided by the precue predict RTs. This would suggest that trial-by-trial CNV amplitude variations are related to response preparation, no matter what drives these fluctuations.

Because we have no exact individual electrode localizations available, an additional source localization analysis would not have provided new insights beyond our previous study (Scheibe et al., 2009).

fMRI data analysis. Preprocessing and voxel-based statistical analysis of the imaging data was done within the framework of the general linear model (Friston et al., 1995; Kiebel and Holmes, 2003; Friston, 2005), as implemented in SPM2 (Statistical Parametrical Mapping, Wellcome Department of Cognitive Neurology, University College London, London UK; http://www.fil.ion.ucl.ac.uk/spm). Preprocessing included slicetime correction, realignment to correct for movement artifacts, normalization to the Montreal Neurological Institute (MNI) standard brain, and spatial smoothing with a three-dimensional Gaussian filter of $6 \mathrm{~mm}$ full-width half-maximum using standard SPM2 methods.

Global linear trends were minimized through high-pass filtering of the data with a cutoff period of $128 \mathrm{~s}$, and intrinsic autocorrelations were modeled. Design matrices were generated using event-related regressors convolved with canonical hemodynamic response functions.

In the first model (model 1), we assessed activity related to the processing of PP during the foreperiod. We specified a model consisting of eight regressors representing the precue S1 of the conditions PP 1.0, PP 0.75 , and PP 0.5 , followed by left and right responses, respectively, and additional left and right button presses. We modeled the condition regressors for left and right preparation separately to enable detection of lateralized preparatory activity during the foreperiod. The regressors for the conditions comprised the duration of the foreperiod, starting with presentation of the precue and lasting $2000 \mathrm{~ms}$. The regressors for the button presses started with the button press and were modeled as stick functions. Additionally, we included the movement parameters (as identified by the realignment procedure) into the model as covariates of no interest to account for task-unrelated additional variance. To obtain group activation maps, contrast images were computed for each participant for PP $1.0>$ PP 0.75 and PP $0.75>$ PP 0.5 containing left- and right-hand response trials and for PP $1.0>$ PP 0.75 and PP $0.75>$ PP 0.5 containing only left- or right-hand responses, respectively, using $t$ statistics. To identify voxels with BOLD signals increasing parametrically with increasing PP, we calculated a group-level conjunction analysis containing the subject-level contrast images PP $1.0>$ PP 0.75 and PP $0.75>$ PP 0.5 . We searched for voxels that were significantly active in both contrasts (Nichols et al., 2005). To detect lateralized activity, we conducted second and third conjunction analyses with the corresponding contrasts containing only left- or right-hand responses, respectively. The significance threshold for the conjunction analyses was set to $t \geq 2.75$ ( $p<0.005$, 
uncorrected for multiple comparisons, cluster size threshold of 10 voxels).

We additionally extracted the percentage signal change related to the foreperiod regressors PP 0.5, PP 0.75, and PP 1.0 in the region revealed by the conjunction analysis with responses from both hands. Percentage signal change was compared between conditions using a repeated-measures ANOVA with the factor PP condition (PP 0.5, PP 0.75, and PP 1.0) and post hoc paired $t$ tests.

To identify voxels with BOLD signals covarying with the $\mathrm{CNV}$ amplitude per trial, we specified the second model (model 2) with one regressor containing the precue onsets with a duration of $2000 \mathrm{~ms}$ and a respective parametric regressor containing the single-trial $\mathrm{CNV}$ amplitude. We also included the movement parameters into the model as covariates of no interest to account for task-unrelated additional variance. Contrast images were computed for the regressor CNV amplitude as main contrast using $t$ statistics. The contrast images were entered into mixed-effects analyses to obtain group activation. The significance threshold was set to $t \geq 2.95$ ( $p<0.005$, uncorrected for multiple comparisons, cluster size threshold of 10 voxels). This relatively liberal threshold is comparable with previous studies (Debener et al., 2005, Eichele et al., 2005; Mulert et al., 2008) and ensures a low likelihood of false negatives (Goldman et al., 2009).

The variability of the CNV amplitude is based on the influence of PP information as well as other processes (e.g., attention) occurring during the preparatory interval. To separate the proportion of variance attributable to PP, we calculated a regression of the vector with the single-trial CNV amplitude and the vector with PP for each trial ( 3 for PP 1.0, 2 for PP 0.75, and 1 for PP 0.5). The resulting residual vector excluded the proportion of variance that is attributable to PP. To identify voxels covarying with the single-trial CNV amplitude when the PP contribution is ruled out, the third model (model 3) was specified. Model 3 was built of one regressor containing the precue onsets with a duration of $2000 \mathrm{~ms}$ and a respective parametric regressor containing the residuals for each trial. We also included the movement parameters into the model as covariates of no interest to account for task-unrelated additional variance. The onset regressor and the covariates were identical to model 2. Contrast images were computed with the residuals regressor as the main contrast using $t$ statistics and mixed-effects analyses to obtain group activation. The significance threshold was set to $t \geq 2.95(p<0.005$, uncorrected for multiple comparisons, cluster size threshold of 10 voxels). By comparing the results of model 2 and model 3, we identified voxels whose activity covaried with the CNV and was attributable only to $\mathrm{PP}$ processing and not to general preparation mechanisms. To depict regions in which BOLD signal is attributed exclusively to PP processing, activated voxels of model 2 and model 3 were projected to the same template brain. Regions that are only activated in model 2 were attributed to PP contribution, whereas regions that are only activated in model 3 are not involved in PP integration.

\section{Results}

\section{Behavioral results}

The mean RTs for each condition are depicted in Figure $1 B$ (top). The analysis revealed a significant effect for PP condition $\left(F_{(3,45)}=68.1, p<0.0001\right)$. RTs decreased with increasing $\mathrm{PP}$ and differed significantly between all conditions $\left(t_{(15)}>3.6, p<0.05\right)$. Mean RTs were $509.0 \mathrm{~ms}$ for PP $0.75-$ invalid, $475.2 \mathrm{~ms}$ for PP $0.5,433.4 \mathrm{~ms}$ for PP 0.75 -valid, and $411.8 \mathrm{~ms}$ for PP 1.0. Mean error rates were 0.09 for PP 0.75 -
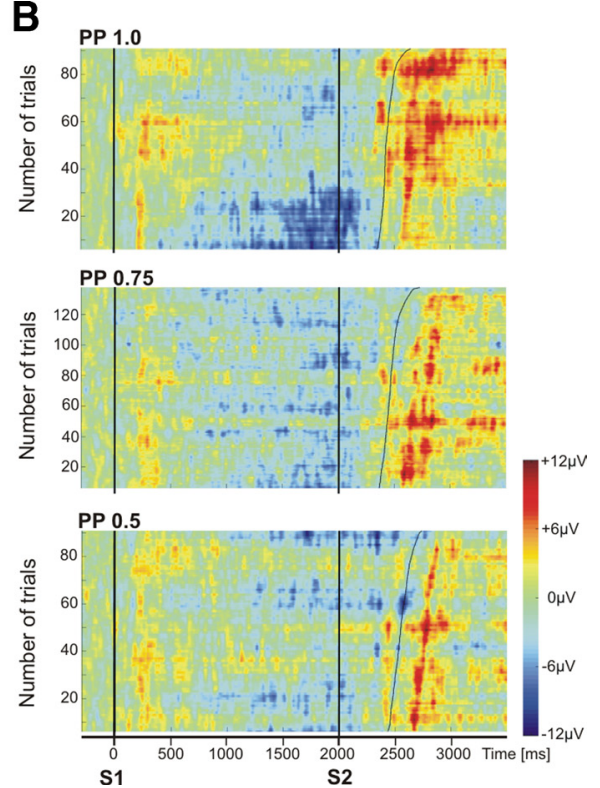

s1

Figure 2. Mean CNV amplitude and single trials. $\boldsymbol{A}$, Mean CNV amplitudes. Time course (top) and amplitude (bottom) of the CNV at electrode position C $z$ for the conditions PP 0.75, PP 0.5, and PP 1.0. The CNV increased parametrically with increasing PP. $\boldsymbol{B}$ Single-trial data. Single-trial data of one exemplary participant. Trials are depicted separately for conditions PP 0.75, PP 0.5, and PP 1.0 and sorted by RT (sigmoid black line). All conditions show a considerable trial-to-trial variability in the CNV amplitude.

invalid, 0.02 for PP 0.5, 0.02 for PP 0.75-valid, and 0.03 for PP 1.0. Because the error rates were rather low, errors were not analyzed further.

The analysis of the vincentized cumulative probability distributions of RTs for all PP conditions revealed that the effect of PP was clearly present throughout the RT distribution, with the exception of the slowest decile (Fig. $1 B$, bottom). We found main effects for $\operatorname{PP}\left(F_{(3,45)}=43.0, p<0.0001\right)$ and decile $\left(F_{(9,135)}=\right.$ $177.8, p<0.0001)$, as well as an interaction between these factors $\left(F_{(27,405)}=4.2, p<0.0001\right)$.

\section{EEG results}

The mean CNV per condition at electrode position $\mathrm{Cz}$ is shown in Figure $2 \mathrm{~A}$. Increases in PP elicited an increasingly negative CNV amplitude. The analysis revealed main effects of PP condition $\left(F_{(2,4)}=8.9, p<0.05\right)$ and electrode $\left(F_{(46,92)}=\right.$ $4.4, p<0.0001)$, as well as an interaction between these factors $\left(F_{(92,184)}=2.3, p<0.0001\right)$. The CNV was maximal at the centromedial electrode position $\mathrm{Cz}$. The subsequent ANOVA at this electrode position revealed a main effect for PP condition $\left(F_{(2,30)}=16.6, p<0.0001\right)$. Post hoc pairwise $t$ tests comparing the $\mathrm{CNV}$ at $\mathrm{Cz}$ between the conditions revealed significant differences in all relevant comparisons $\left(t_{(15)}>2.3\right.$, $p<0.05$, Bonferroni's corrected). Single-trial ERPs sorted by $\mathrm{RT}$ in the various conditions are depicted for one exemplary participant in Figure $2 B$, demonstrating considerable trial-totrial variability of the $\mathrm{CNV}$ amplitude within each condition.

We examined the relationship of the single-trial CNV amplitudes to behavior. Individual correlation coefficients per participant were significant in half of the sample. The $t$ test showed that high CNV amplitudes were associated with short RTs when using single-trial CNV amplitudes $\left(r=-0.08, t_{(15)}=\right.$ $-4.2, p<0.001$ ), as well as when using the residuals of the single-trial CNV amplitudes after removal of the PP effect ( $r=$ $\left.-0.04, t_{(15)}=-2.0, p<0.05\right)$. 
A
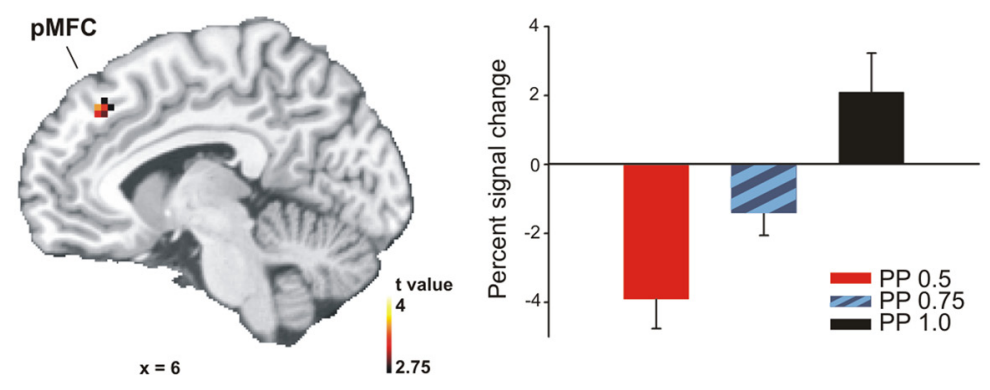

B
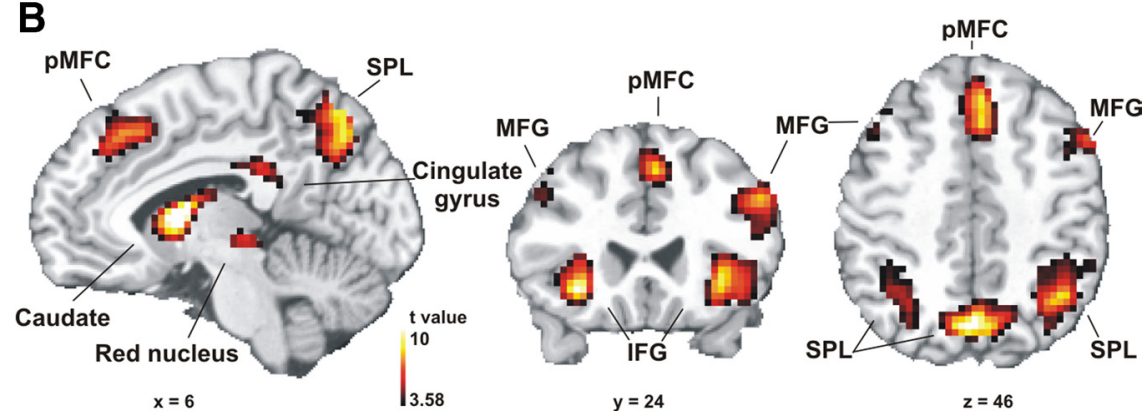

Figure 3. $f M R I$ results of the conjunction analysis. $\boldsymbol{A}$, Conjunction analysis. The conjunction analysis (model 1 ) designed to identify voxels parametrically modulated by PP revealed activation in the PMFC. The percentage signal changes for each condition are depicted on the right. Neural activation (shown at $p<0.005$, uncorrected) is projected on an MNI template (Colin). $\boldsymbol{B}$, Contrast PP $1.0>0.5$. The contrast PP $1.0>0.5$ activated regions in the PMFC, MFG, IFG, superior parietal lobule (SPL), cingulate gyrus, and midbrain structures. Activation clusters (shown at $p<0.001$, uncorrected) are projected on an MNI template (Colin).

\section{EEG / fMRI results}

Figure $4 A$ shows the activation pattern covarying with the $\mathrm{CNV}$ amplitude, as revealed by model 2. Increasing $\mathrm{CNV}$ amplitude correlated with increasing activity in several prefrontal regions. We found BOLD signal covarying with CNV amplitude in the right middle frontal gyrus (MFG)/superior prefrontal sulcus (BA $6)$, the left inferior frontal gyrus (IFG)/ inferior prefrontal sulcus (BA 9), the left superior frontal gyrus (SFG) (BA 6), the right IFG (BA 44/45), and the dorsolateral prefrontal cortex (DLPFC) in the left MFG (BA 46). We also observed increased activation bilaterally in the inferior parietal lobule (IPL) (BA 40). We found bilateral activation in the putamen in the dorsal striatum. Table 2 contains the activation peaks for the results of the regions, in which the BOLD signal covaried with the CNV amplitude.

Figure $4 B$ depicts the overlapping localization of activations revealed by model 2 with the single-trial CNV amplitude regressor and model 3 with the residuals regressor of the single-trial $\mathrm{CNV}$ amplitude. Activations covarying with the CNV amplitude that include the contribution of PP are shown in blue. Activations covarying with the CNV amplitude

\section{fMRI results}

Figure $3 A$ shows the result of the conjunction analysis (model 1) related to the preparation period during which the probability precue was delivered. The conjunction analysis contained the contrasts PP $1.0>$ PP 0.75 and PP $0.75>$ PP 0.5 and searched for voxels that were significantly active in both contrasts. The BOLD signal in a region in the posterior medial frontal cortex (pMFC) increased parametrically with increasing PP. The activated region is located in the border zone between Brodmann areas (BA) 8, 6, and 32. Percentage signal change for each condition is depicted in Figure $3 A$ (right). The negativity of the signal change reflects relative deviations from the implicit baseline in the model rather than deactivations or negative BOLD signals. The analysis revealed a significant effect for PP condition $\left(F_{(2,30)}=23.0, p<\right.$ $0.0001)$. Percentage signal change increased with increasing PP and differed significantly across all conditions $\left(t_{(15)}>4.1, p<\right.$ $0.005)$. The additional conjunction analyses containing the contrasts PP $1.0>$ PP 0.75 and PP $0.75>$ PP 0.5 for left- and rightresponse trials revealed the same activated region when the cluster size threshold was reduced to four voxels. We did not find lateralized motor activity. Table 1 contains the activation peaks for the results of the conjunction analysis.

Whereas the conjunction analysis above focuses on the increasing information content of the precue associated with increasing PP, Figure $3 B$ shows the results of the direct contrast PP $1.0>$ PP 0.5 revealing a more widespread activation pattern associated with the additional motor preparation processes occurring at $100 \%$ certainty (PP 1.0). We have shown previously that only PP 1.0 elicits a lateralized readiness potential (Scheibe et al., 2009), suggesting that effector-specific motor preparation processes take place in this condition. Table 1 contains the activation peaks for the results of the contrast PP $1.0>$ PP 0.5 . residuals, when PP contribution is partialled out, are shown in red. Overlapping activations are shown in green. Blue regions can be interpreted as being activated solely through trial-to-trial fluctuations based on PP processing. The DLPFC in the left MFG (BA 46), the right IFG (BA 44/45), and the right IPL were activated exclusively as a result of the PP-driven CNV amplitude modulation. Table 3 contains the activation peaks of the regions in which the BOLD signal covaried with the CNV amplitude residuals.

\section{Discussion}

The goal of the present study was to shed light on the neural basis of PP integration into the decision-making process and associated preparatory processes. First, we used a conventional fMRI analysis approach to identify brain regions in which neural activity is parametrically modulated by PP. Second, accounting for trial-to-trial variations in PP processing, we applied an EEGinformed $\mathrm{fMRI}$ analysis by incorporating the single-trial CNV amplitude into the fMRI model as a regressor. This enabled us to investigate regions that represent the trial-to-trial fluctuations of $\mathrm{PP}$ processing reflected by the CNV.

At the behavioral level, we found a robust parametrical effect of PP, with RT decreasing when PP increased. This effect was not attributable to a small set of trials for which participants prepared intensely but rather occurred in a substantial number of trials. This finding demonstrates that participants used the probabilitybased advance information to optimize their behavior by preparing a response. Decreasing reaction time with increasing prior probability was also found in oddball paradigms (Bénar et al., 2007; Goldman et al., 2009), with rare target stimuli occurring with low probability and frequent distractor stimuli occurring with high probability. However, in contrast to our task, prior 
Table 1. Anatomical locations and MNI coordinates for the results of the conjunction analyses and the regions activated in contrast PP $1.0>$ PP 0.5 (model 1 )

\begin{tabular}{|c|c|c|c|c|c|c|c|c|}
\hline & & & & & $\mathrm{MNICC}$ & & & \\
\hline & Cluster size & Anatomical region & BA & Hemisphere & $x$ & $y$ & $z$ & Voxel $t$ value \\
\hline Conjunction & 15 & pMFC & $6 / 8$ & $\mathrm{R}$ & 6 & 36 & 42 & 3.51 \\
\hline Conjunction left responses & 6 & pMFC & $6 / 8$ & $\mathrm{R}$ & 9 & 33 & 42 & 3.23 \\
\hline Conjunction right responses & 4 & pMFC & $6 / 8$ & $\mathrm{R}$ & 3 & 36 & 45 & 3.07 \\
\hline PP $1.0>$ PP 0.5 & 160 & MFG & 9 & L & -39 & 12 & 30 & 8.61 \\
\hline & 127 & IFG & 47 & $\mathrm{R}$ & 33 & 27 & -6 & 8.22 \\
\hline & 209 & SPL & 7 & L & -27 & 75 & 48 & 8.01 \\
\hline & 111 & IFG & 47 & L & -33 & 21 & -9 & 7.31 \\
\hline & 158 & pMFC & $6 / 8$ & $\mathrm{R}$ & 6 & 21 & 48 & 6.66 \\
\hline & 112 & Cingulate gyrus & 31 & $\mathrm{R}$ & 3 & -33 & 30 & 6.65 \\
\hline & 692 & SPL & 7 & $\mathrm{R}$ & 9 & -69 & 57 & 6.61 \\
\hline & 282 & MFG & 9 & $\mathrm{R}$ & 51 & 27 & 27 & 6.49 \\
\hline & 102 & Cerebellum & & L & -12 & -72 & -30 & 6.20 \\
\hline & 57 & MFG & 10 & L & -33 & 51 & -6 & 6.09 \\
\hline & 88 & Fusiform gyrus & 37 & $\mathrm{R}$ & 48 & -57 & -15 & 6.02 \\
\hline & 148 & ITG & 19 & L & -54 & -63 & -18 & 5.88 \\
\hline & 55 & MFG & 10 & $\mathrm{R}$ & 27 & 60 & 9 & 5.70 \\
\hline & 58 & Caudate & & $\mathrm{R}$ & 9 & 9 & 6 & 5.69 \\
\hline & 40 & MFG & 46 & L & -45 & 36 & 21 & 5.36 \\
\hline & 76 & Caudate & & L & -9 & 9 & 6 & 5.34 \\
\hline & 27 & Red nucleus & & $\mathrm{R}$ & 3 & -24 & -3 & 5.22 \\
\hline & 84 & MFG & 8 & $\mathrm{R}$ & 27 & 15 & 48 & 5.03 \\
\hline & 22 & MTG & 39 & $\mathrm{R}$ & 36 & -69 & 21 & 4.43 \\
\hline & 10 & SFG & 6 & L & -15 & 9 & 63 & 4.35 \\
\hline & 10 & Subthalamic nucleus & & L & -6 & -12 & -6 & 4.32 \\
\hline & 11 & MFG & 10 & L & -42 & 48 & 18 & 4.22 \\
\hline
\end{tabular}

Conjunction analyses: $p<0.005$, uncorrected; cluster size threshold, 10 voxels. Contrast PP 1.0 > PP 0.5: $p<0.001$, uncorrected; cluster size threshold, 10 voxels. SPL, Superior parietal lobule; ITG, inferior temporal gyrus; MTG, middle temporal gyrus.

probability is manipulated indirectly and implicitly through modulating the time interval between the rare events.

Analogously to RT, the mean CNV amplitude was parametrically modulated by PP and increased from PP 0.5 to PP 1.0. This confirms that a precue containing gradual PP information facilitates premotor preparation, as shown in a previous EEG study (Scheibe et al., 2009). Notably, the PP effect on the CNV amplitude could be replicated even in the hostile scanner environment in which the EEG signal is contaminated by serious artifacts that result from gradient switching and ballistocardiograms (Niazy et al., 2005; Debener et al., 2008).

A conventional fMRI conjunction analysis revealed an area in the posterior part of the MFC in which the BOLD response increased parametrically with increasing PP. This area is located in the border zone between BA 6,8 , and 32 and is commonly referred to as the rostral cingulate zone (RCZ) (Picard and Strick, 2001; Ridderinkhof et al., 2004). In a previous EEG study using an identical task, we found a dipole in the same region during the foreperiod; activity of this dipole was also parametrically modulated by PP (Scheibe et al., 2009).

The functional role of the pMFC is frequently described as performance monitoring and cognitive control signaling the need for behavioral adjustments (Ridderinkhof et al., 2004;

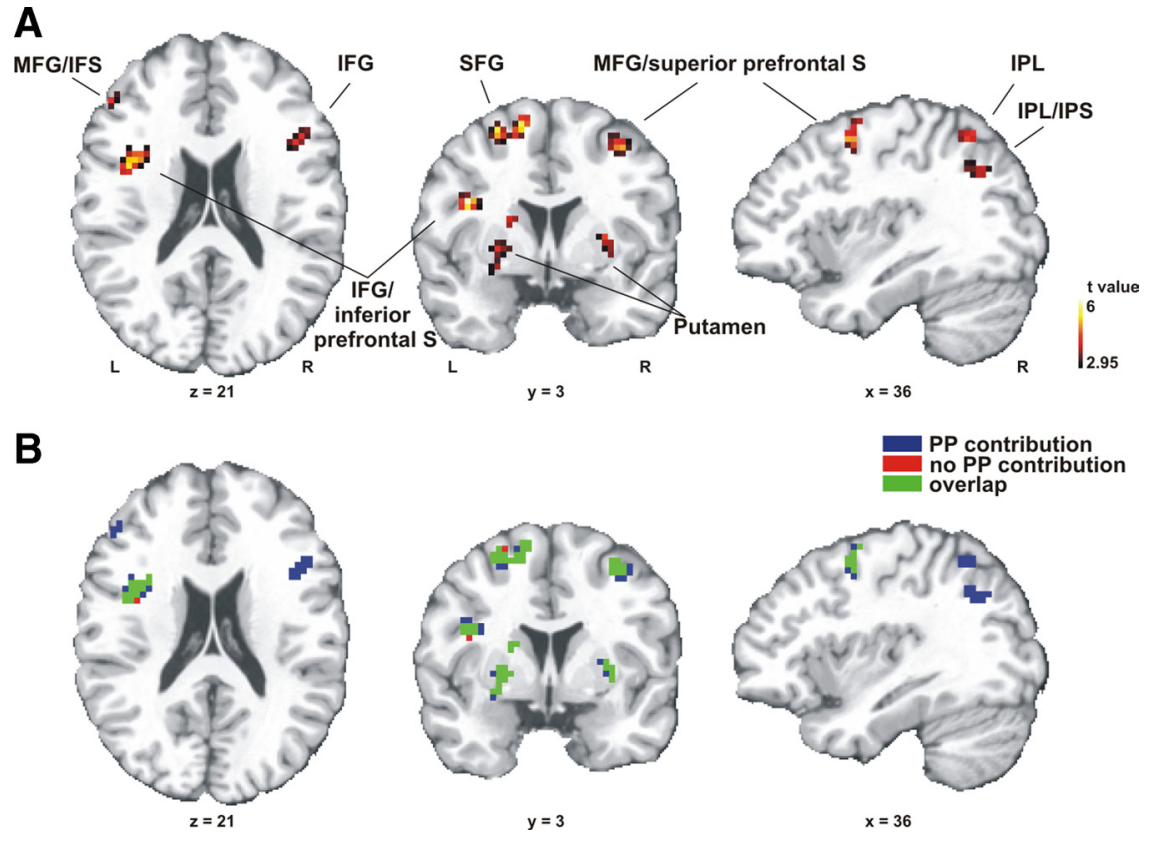

Figure 4. fMRI results of the single-trial analysis. $A$, The BOLD signal covaried with the single-trial CNV amplitude in a set of regions including prefrontal, parietal, and striatal regions (model 2). $\boldsymbol{B}$, This shows differentially activated regions in which BOLD signal covaried with CNV amplitude (model 2, blue), differentially activated regions in which BOLD signal covaried with CNV amplitude residuals (model 3, red), and the overlap between the two models (green). The middle frontal gyrus (i.e., DLPFC), the IFG, and the IPL represent the contribution of PP to the trial-to-trial fluctuations of the CNV amplitude. Activation clusters (shown at $p<0.005$, uncorrected) are projected on an MNI template (Colin). IPS, Inferior parietal sulcus; L, left; $\mathrm{R}$, right; $S$, sulcus.

Ullsperger and von Cramon, 2004b; Heekeren et al., 2008). This description fits with the parametrical activation in this region because the probability precue task requires monitoring concerning behavioral adjustment during the foreperiod. Preparation processes were initiated depending on the probability informa- 
Table 2. Anatomical locations and MNI coordinates for the results of the regions covarying with the single-trial CNV amplitude (model 2)

\begin{tabular}{|c|c|c|c|c|c|c|c|c|}
\hline & \multirow[b]{2}{*}{ Cluster size } & \multirow[b]{2}{*}{ Anatomical region } & \multirow[b]{2}{*}{$\mathrm{BA}$} & \multirow[b]{2}{*}{ Hemisphere } & \multicolumn{3}{|c|}{ MNI coordinates } & \multirow[b]{2}{*}{ Voxel $t$ value } \\
\hline & & & & & $x$ & $y$ & $Z$ & \\
\hline \multirow[t]{11}{*}{ CNV amplitude } & 39 & Posterior MFG, superior prefrontal S & 6 & $\mathrm{R}$ & 33 & 0 & 54 & 6.06 \\
\hline & 43 & IFG, inferior prefrontal S & 9 & $\mathrm{~L}$ & -42 & 3 & 24 & 5.91 \\
\hline & 61 & SFG & 6 & $\mathrm{~L}$ & -12 & 6 & 63 & 5.56 \\
\hline & 18 & IFG & $44 / 45$ & $\mathrm{R}$ & 45 & 18 & 18 & 4.90 \\
\hline & 33 & Putamen & & $\mathrm{R}$ & 30 & 6 & -3 & 4.70 \\
\hline & 29 & IPL, IPS & & $\mathrm{R}$ & 30 & -39 & 42 & 4.62 \\
\hline & 18 & $\mathrm{IPL}$ & 40 & $\mathrm{R}$ & 36 & -57 & 51 & 4.32 \\
\hline & 46 & IPL & 40 & $\mathrm{~L}$ & -45 & -30 & 48 & 4.25 \\
\hline & 69 & Putamen & & $\mathrm{L}$ & -21 & 6 & 12 & 4.13 \\
\hline & 10 & MFG, IFS & 46 & $\mathrm{~L}$ & -51 & 36 & 21 & 4.12 \\
\hline & 16 & IPL, IPS & 39 & $\mathrm{R}$ & 36 & -66 & 36 & 3.88 \\
\hline
\end{tabular}

$p<0.005$, uncorrected; cluster size threshold, 10 voxels. S, Sulcus; IPS, inferior parietal sulcus; IFS, inferior frontal sulcus.

Table 3. Anatomical locations and MNI coordinates for the regions covarying with the single-trial CNV amplitude residuals (model 3)

\begin{tabular}{|c|c|c|c|c|c|c|c|c|}
\hline & \multirow[b]{2}{*}{ Cluster size } & \multirow[b]{2}{*}{ Anatomical region } & \multirow[b]{2}{*}{$\mathrm{BA}$} & \multirow[b]{2}{*}{ Hemisphere } & \multicolumn{3}{|c|}{ MNI coordinates } & \multirow[b]{2}{*}{ Voxel $t$ value } \\
\hline & & & & & $x$ & $y$ & $z$ & \\
\hline \multirow[t]{7}{*}{ CNV amplitude residuals } & 58 & SFG & 6 & $\mathrm{~L}$ & -12 & 6 & 63 & 5.73 \\
\hline & 31 & IFG & 9 & $\mathrm{~L}$ & -42 & 3 & 21 & 5.58 \\
\hline & 31 & Posterior MFG, superior prefrontal S & 6 & $\mathrm{R}$ & 33 & 0 & 54 & 5.14 \\
\hline & 61 & Putamen & & $\mathrm{L}$ & -27 & 0 & 0 & 4.92 \\
\hline & 12 & IPL & 40 & $\mathrm{~L}$ & -45 & -30 & 48 & 4.55 \\
\hline & 28 & Putamen & & $\mathrm{R}$ & 30 & 6 & -3 & 4.37 \\
\hline & 21 & IPL & & $\mathrm{R}$ & 30 & -33 & 45 & 4.20 \\
\hline
\end{tabular}

$p<0.005$, uncorrected; cluster size threshold, 10 voxels. S, Sulcus.

tion delivered by the precue. Knowledge of the PP enables performance to be adjusted to optimize the achievement of action goals. If the probability is high for one response side (PP 1.0), then preparatory processes must be initiated to perform with maximum efficiency. If the probability is low (PP 0.5), there is no need for behavioral adjustment because any action can only be initiated when more information is available. In accordance with our results, the RCZ shows a stronger response when the need for behavioral adjustment is high (indicated by a high informative content of negative feedback) than when negative feedback is less informative (Jocham et al., 2009). This supports the hypothesis that activity in the pMFC reflects the degree to which the PP information of the precue is used to guide subsequent decisions. In a similar vein, Rushworth and colleagues (Rushworth et al., 2004, 2007; Rushworth, 2008) postulate that the MFC has a central role in action selection and response preparation. They state that this region does not select individual actions but instead selects superordinate sets of action selection rules and is involved in anticipatory preparation. MFC activity is enhanced in tasks that include conflict (Botvinick et al., 2001; Carter and van Veen, 2007), decision uncertainty (Volz et al., 2005), and error processing (Holroyd and Coles, 2002; Ullsperger and von Cramon, 2004a). Like our task, these conditions demand enhancement of cognitive control and performance monitoring.

In summary, the pMFC is active in a variety of tasks and contexts. Its role seems to be best described as signaling other brain regions that a behavioral change is needed to regulate task performance in an adaptive manner, thereby integrating information from previous knowledge or experience. In our task, probability information was parametrically encoded in the pMFC, which resulted in enhancement of preparatory activity. This suggests that the pMFC is also involved in the integration of probability information.

In everyday behavior, however, environmental conditions and demands as well as intrinsic brain activity vary from situation to situation. Likewise, the processing of probability information, which is available before making a decision, is affected by many factors that change dynamically over time. In each trial of our task, a unique pattern of interacting processes occurs. For example, the instantaneous attentional state has an influence on the perception and processing of the probability information of the actual precue. This influences expectations of the subsequent stimulus, and, consequently, preparatory processes are executed to a greater or lesser extent. The trial-to-trial fluctuations in preparation-related brain activity are reflected in ERP components. Recently, it has been shown that the trial-by-trial variability of the P3 amplitude is not random noise but can be better explained by a formal model of cognitive factors fluctuating from trial to trial (Mars et al., 2008). The CNV represents at least partly the processing of probability information and preparatory processes, because the amplitude is parametrically modulated by PP (Scheibe et al., 2009) and was related to RT on a single-trial basis. Our aim was to identify brain regions associated with processes involved in PP integration and preparation that fluctuate from trial to trial. Because trial-to-trial fluctuations are not considered with a conventional fMRI approach, we took the additional step of integrating the single-trial CNV amplitudes with the fMRI analysis, as pioneered by Nagai et al. (2004). This allowed us to map activity associated with trial-to-trial fluctuations in PP integration and general preparatory processes. To separate the contributions of PP processing from general preparatory processes and unspecific ongoing fluctuations, we conducted an additional analysis and controlled for contributions of PP processing.

We found a set of regions of mainly frontal, parietal, and striatal regions representing the trial-to-trial fluctuations in CNV amplitude during the preparation period in which PP information is processed. A subset of these regions, namely, the left DLPFC, the right IFG, and the right IPL, showed activations that exclusively represent the contributions of PP to CNV amplitude fluctuations. 
Response preparation in general involves several parts of a frontoparietal network and the basal ganglia (Thoenissen et al., 2002; Toni et al., 2002; Elsinger et al., 2006). In frontal premotor regions and the inferior parietal lobe, preparatory activity is sensitive to previous nonprobabilistic information about the subsequent event and the required response (Toni et al., 2001; Thoenissen et al., 2002; Klaver et al., 2004). The putamen also plays a role in unspecific preparation mechanisms and planning behavior (Monchi et al., 2006). Thus, the activations of frontal, parietal, and striatal regions that fluctuate together with the CNV amplitude probably represent general response preparation processes that are independent of the PP information.

In contrast, we found activations in the DLPFC, IFG, and IPL exclusively related to PP-induced CNV fluctuations, indicating that those regions are involved in the integration of PP information for response preparation on a trial-to-trial basis. A very similar configuration of activated areas in the dorsal frontal and parietal cortex is known to be involved in cue processing in anticipation of a stimulus, regardless of the modality of the cue (Corbetta and Shulman, 2002; Peelen et al., 2004). Lateral prefrontal regions regulate the current focus of attention through their interaction with parietal areas. The DLPFC contributes selectively to the establishment of an attentional set for taskrelevant information during cue processing (Weissman et al., 2003). Other authors state that the left DLPFC is engaged during the preparation period by regulating and actively maintaining the attentional demands of the task (MacDonald et al., 2000). Activity fluctuation in these areas may therefore represent trial-specific attentional effort, depending on the probability information. In line with the finding that DLPFC activity correlated directly with fluctuating PP processing, single-unit recordings in monkeys revealed that the firing rate of neurons in the DLPFC increases with increasing PP of the subsequent response (Quintana and Fuster, 1999). Although the prefrontal cortex plays an important role in attentional executive control, parietal regions are recruited when stimulus-response associations can be prepared in advance (Corbetta and Shulman, 2002). Primate studies with twoalternative forced-choice tasks have shown that neurons in the lateral intraparietal area integrate information from multiple sources and encode the likelihood ratio concerning the subsequent selection between the two decision alternatives (Gold and Shadlen, 2001; Sugrue et al., 2005). Thus, fluctuations of activation in parietal regions may be attributed to the integration of information from different sources, including PP information when planning the upcoming response.

The CNV seems to reflect response preparation processes and thus covaries with PP. Fluctuations of PP processing that do not influence the speed or accuracy of the response should thus not be reflected in the $\mathrm{CNV}$ either. The functional role of each single region covarying with the $\mathrm{CNV}$ amplitude and their causal connections need to be elucidated in additional research.

It may appear surprising that the results of the conventional and the single-trial EEG-informed fMRI analyses differ quite substantially. These results reveal that both analyses address different functional aspects of PP processing in decision making and response selection. Whereas the conjunction analysis focuses on the mere effect of information content associated with increasing $\mathrm{PP}$ and neglects trial-by-trial fluctuations, the single-trial CNV quantification elucidates the fluctuating influence of PP on response preparation. This is supported by the significant relationship of CNV amplitude and RT. In other words, trial-by-trial variations of subjective PP perception as well as fluctuations in the transfer of this information to the response selection stage find their correlate in the CNV and the associated hemodynamic activity.

In summary, the integration of PP information into the decision-making process relies on dynamic adjustments of cognitive control during the foreperiod. It engages a distributed set of brain regions that interact closely, but it nevertheless consists of functionally different components (MacDonald et al., 2000). Within this system, the pMFC is likely to be involved in evaluative processes and performance monitoring that signals the need for behavioral adjustment and initiates it. Probability information enhanced activity in this region in a parametric manner regardless of trial-by-trial fluctuations of brain state or attentional situation, signaling the need for response preparation. Frontal and parietal regions as well as the striatum are involved in single-trial preparation processes. The DLPFC, the IFG, and the IPL showed activations that exclusively represented the trial-to-trial processing and integration of PP. The data suggest that the integration of PP information into the decision-making process both includes and depends on several processes that are only distinguishable by a trial-to-trial-based analysis of simultaneously recorded EEG/fMRI.

\section{References}

Bell AJ, Sejnowski TJ (1995) An information-maximization approach to blind separation and blind deconvolution. Neural Comput 7:1129-1159.

Bénar CG, Schön D, Grimault S, Nazarian B, Burle B, Roth M, Badier JM, Marquis P, Liegeois-Chauvel C, Anton JL (2007) Single-trial analysis of oddball event-related potentials in simultaneous EEG-fMRI. Hum Brain Mapp 28:602-613.

Botvinick MM, Braver TS, Barch DM, Carter CS, Cohen JD (2001) Conflict monitoring and cognitive control. Psychol Rev 108:624-652.

Carpenter RH (2004) Contrast, probability, and saccadic latency; evidence for independence of detection and decision. Curr Biol 14:1576-1580.

Carpenter RH, Williams ML (1995) Neural computation of log likelihood in control of saccadic eye movements. Nature 377:59-62.

Carter CS, van Veen V (2007) Anterior cingulate cortex and conflict detection: an update of theory and data. Cogn Affect Behav Neurosci 7:367-379.

Corbetta M, Shulman GL (2002) Control of goal-directed and stimulusdriven attention in the brain. Nat Rev Neurosci 3:201-215.

Debener S, Ullsperger M, Siegel M, Fiehler K, von Cramon DY, Engel AK (2005) Trial-by-trial coupling of concurrent electroencephalogram and functional magnetic resonance imaging identifies the dynamics of performance monitoring. J Neurosci 25:11730-11737.

Debener S, Ullsperger M, Siegel M, Engel AK (2006) Single-trial EEG-fMRI reveals the dynamics of cognitive function. Trends Cogn Sci 10:558-563.

Debener S, Mullinger KJ, Niazy RK, Bowtell RW (2008) Properties of the ballistocardiogram artefact as revealed by EEG recordings at 1.5, 3 and $7 \mathrm{~T}$ static magnetic field strength. Int J Psychophysiol 67:189-199.

Delorme A, Makeig S (2004) EEGLAB: an open source toolbox for analysis of single-trial EEG dynamics including independent component analysis. J Neurosci Methods 134:9-21.

Delorme A, Sejnowski T, Makeig S (2007) Enhanced detection of artifacts in EEG data using higher-order statistics and independent component analysis. Neuroimage 34:1443-1449.

Eichele T, Specht K, Moosmann M, Jongsma ML, Quiroga RQ, Nordby H, Hugdahl K (2005) Assessing the spatiotemporal evolution of neuronal activation with single-trial event-related potentials and functional MRI. Proc Natl Acad Sci U S A 102:17798-17803.

Elsinger CL, Harrington DL, Rao SM (2006) From preparation to online control: reappraisal of neural circuitry mediating internally generated and externally guided actions. Neuroimage 31:1177-1187.

Esposito F, Mulert C, Goebel R (2009) Combined distributed source and single-trial EEG-fMRI modeling: application to effortful decision making processes. Neuroimage 47:112-121.

Fox MD, Snyder AZ, Zacks JM, Raichle ME (2006) Coherent spontaneous activity accounts for trial-to-trial variability in human evoked brain responses. Nat Neurosci 9:23-25. 
Friston KJ (2005) Models of brain function in neuroimaging. Annu Rev Psychol 56:57-87.

Friston KJ, Holmes AP, Worsley KJ, Poline J-P, Frith CD, Frackowiak RSJ (1995) Statistical parametric maps in functional imaging: a general linear approach. Hum Brain Mapp 2:189-210.

Glimcher PW (2001) Making choices: the neurophysiology of visualsaccadic decision making. Trends Neurosci 24:654-659.

Gold JI, Shadlen MN (2001) Neural computations that underlie decisions about sensory stimuli. Trends Cogn Sci 5:10-16.

Gold JI, Shadlen MN (2007) The neural basis of decision making. Annu Rev Neurosci 30:535-574.

Goldman RI, Wei CY, Philiastides MG, Gerson AD, Friedman D, Brown TR, Sajda P (2009) Single-trial discrimination for integrating simultaneous EEG and fMRI: identifying cortical areas contributing to trial-to-trial variability in the auditory oddball task. Neuroimage 47:136-147.

Heekeren HR, Marrett S, Bandettini PA, Ungerleider LG (2004) A general mechanism for perceptual decision-making in the human brain. Nature 431:859-862.

Heekeren HR, Marrett S, Ungerleider LG (2008) The neural systems that mediate human perceptual decision making. Nat Rev Neurosci 9:467-479.

Holroyd CB, Coles MG (2002) The neural basis of human error processing: reinforcement learning, dopamine, and the error-related negativity. Psychol Rev 109:679-709.

Jocham G, Neumann J, Klein TA, Danielmeier C, Ullsperger M (2009) Adaptive coding of action values in the human rostral cingulate zone. J Neurosci 29:7489-7496.

Jung TP, Makeig S, Humphries C, Lee TW, McKeown MJ, Iragui V, Sejnowski TJ (2000a) Removing electroencephalographic artifacts by blind source separation. Psychophysiology 37:163-178.

Jung TP, Makeig S, Westerfield M, Townsend J, Courchesne E, Sejnowski TJ (2000b) Removal of eye activity artifacts from visual event-related potentials in normal and clinical subjects. Clin Neurophysiol 111:1745-1758.

Kiebel SJ, Holmes AP (2003) The general linear model. In: Human brain function (Frackowiak RSJ, Friston KJ, Frith CD, Dolan RJ, Price CJ, Zeki S, Ashburner J, Penny WD, eds). San Diego: Academic.

Klaver P, Fell J, Weis S, De Greiff A, Ruhlmann J, Reul J, Elger CE, Fernández G (2004) Using visual advance information: an event-related functional MRI study. Brain Res Cogn Brain Res 20:242-255.

MacDonald AW 3rd, Cohen JD, Stenger VA, Carter CS (2000) Dissociating the role of the dorsolateral prefrontal and anterior cingulate cortex in cognitive control. Science 288:1835-1838.

Mars RB, Debener S, Gladwin TE, Harrison LM, Haggard P, Rothwell JC, Bestmann S (2008) Trial-by-trial fluctuations in the event-related electroencephalogram reflect dynamic changes in the degree of surprise. J Neurosci 28:12539-12545.

Mazurek ME, Roitman JD, Ditterich J, Shadlen MN (2003) A role for neural integrators in perceptual decision making. Cereb Cortex 13:1257-1269.

Miller J (1998) Effects of stimulus-response probability on choice reaction time: evidence from the lateralized readiness potential. J Exp Psychol 24:1521-1534.

Monchi O, Petrides M, Strafella AP, Worsley KJ, Doyon J (2006) Functional role of the basal ganglia in the planning and execution of actions. Ann Neurol 59:257-264.

Mulert C, Seifert C, Leicht G, Kirsch V, Ertl M, Karch S, Moosmann M, Lutz J, Möller HJ, Hegerl U, Pogarell O, Jäger L (2008) Single-trial coupling of EEG and fMRI reveals the involvement of early anterior cingulate cortex activation in effortful decision making. Neuroimage 42:158-168.

Nagai Y, Critchley HD, Featherstone E, Fenwick PB, Trimble MR, Dolan RJ (2004) Brain activity relating to the contingent negative variation: an fMRI investigation. Neuroimage 21:1232-1241.

Niazy RK, Beckmann CF, Iannetti GD, Brady JM, Smith SM (2005) Removal of FMRI environment artifacts from EEG data using optimal basis sets. Neuroimage 28:720-737.
Nichols T, Brett M, Andersson J, Wager T, Poline JB (2005) Valid conjunction inference with the minimum statistic. Neuroimage 25:653-660.

Oldfield RC (1971) The assessment and analysis of handedness: the Edinburgh inventory. Neuropsychologia 9:97-113.

Opris I, Bruce CJ (2005) Neural circuitry of judgment and decision mechanisms. Brain Res Brain Res Rev 48:509-526.

Peelen MV, Heslenfeld DJ, Theeuwes J (2004) Endogenous and exogenous attention shifts are mediated by the same large-scale neural network. Neuroimage 22:822-830.

Picard N, Strick PL (2001) Imaging the premotor areas. Curr Opin Neurobiol 11:663-672.

Quintana J, Fuster JM (1999) From perception to action: temporal integrative functions of prefrontal and parietal neurons. Cereb Cortex 9:213-221.

Reddi BA, Carpenter RH (2000) The influence of urgency on decision time. Nat Neurosci 3:827-830.

Reddi BA, Asrress KN, Carpenter RH (2003) Accuracy, information, and response time in a saccadic decision task. J Neurophysiol 90:3538-3546.

Ridderinkhof KR, Ullsperger M, Crone EA, Nieuwenhuis S (2004) The role of the medial frontal cortex in cognitive control. Science 306:443-447.

Rushworth MF (2008) Intention, choice, and the medial frontal cortex. Ann N Y Acad Sci 1124:181-207.

Rushworth MF, Walton ME, Kennerley SW, Bannerman DM (2004) Action sets and decisions in the medial frontal cortex. Trends Cogn Sci 8:410-417.

Rushworth MF, Buckley MJ, Behrens TE, Walton ME, Bannerman DM (2007) Functional organization of the medial frontal cortex. Curr Opin Neurobiol 17:220-227.

Scheibe C, Schubert R, Sommer W, Heekeren HR (2009) Electrophysiological evidence for the effect of prior probability on response preparation. Psychophysiology 46:758-770.

Schwarz W, Stein F (1998) On the temporal dynamics of digit comparison processes. J Exp Psychol 24:1275-1293.

Sugrue LP, Corrado GS, Newsome WT (2005) Choosing the greater of two goods: neural currencies for valuation and decision making. Nat Rev Neurosci 6:363-375.

Thoenissen D, Zilles K, Toni I (2002) Differential involvement of parietal and precentral regions in movement preparation and motor intention. J Neurosci 22:9024-9034.

Toni I, Thoenissen D, Zilles K (2001) Movement preparation and motor intention. Neuroimage 14:S110-S117.

Toni I, Shah NJ, Fink GR, Thoenissen D, Passingham RE, Zilles K (2002) Multiple movement representations in the human brain: an event-related fMRI study. J Cogn Neurosci 14:769-784.

Ullsperger M (2010) EEG-informed fMRI analysis. In: Simultaneous EEG and fMRI: recording, analysis, and application (Ullsperger M, Debener S, eds), pp 153-159. New York: Oxford UP.

Ullsperger M, von Cramon DY (2004a) Neuroimaging of performance monitoring: error detection and beyond. Cortex 40:593-604.

Ullsperger M, von Cramon DY (2004b) Decision making, performance and outcome monitoring in frontal cortical areas. Nat Neurosci 7:1173-1174.

Volz KG, Schubotz RI, von Cramon DY (2005) Variants of uncertainty in decision-making and their neural correlates. Brain Res Bull 67:403-412.

Walter WG, Cooper R, Aldridge V, McCallum WC, Winter AL (1964) Contingent negative variation: an electric sign of sensorimotor associationand expectancy in the human brain. Nature 203:380-384.

Weissman DH, Giesbrecht B, Song AW, Mangun GR, Woldorff MG (2003) Conflict monitoring in the human anterior cingulate cortex during selective attention to global and local object features. Neuroimage 19:1361-1368.

Weissman DH, Roberts KC, Visscher KM, Woldorff MG (2006) The neural bases of momentary lapses in attention. Nat Neurosci 9:971-978. 